\title{
The Matching Energy of Random Multipartite Graphs
}

\author{
Caibing Chang ${ }^{1}$, Bo Deng ${ }^{1,2,3, ~ *, ~ H a i z h e n ~ R e n ~}{ }^{1}$, Feng Fu ${ }^{1}$ \\ ${ }^{1}$ School of Mathematics and Statistics, Qinghai Normal University, Xining, China \\ ${ }^{2}$ Key Laboratory of Tibetan Information Processing and Machine Translation, Xining, China \\ ${ }^{3}$ College of Science, Guangdong University of Petrochemical Technology, Maoming, China
}

\section{Email address:}

changcaibing2018@163.com (Caibing Chang),dengbo450@163.com (Bo Deng), haizhenr@126.com (Haizhen Ren)

${ }^{*}$ Corresponding author

\section{To cite this article:}

Caibing Chang, Bo Deng, Haizhen Ren, Feng Fu. The Matching Energy of Random Multipartite Graphs. American Journal of Mathematical and Computer Modelling. Vol. 4, No. 4, 2019, pp. 94-98. doi: 10.11648/j.ajmcm.20190404.12

Received: September 22, 2019; Accepted: December 2, 2019; Published: December 7, 2019

\begin{abstract}
Let $G_{n}$ be a simple graph with n vertices. Gutman and Wagner founded the theory of random graphs, they introduced the matching energy of the graph $G_{n}$, which was defined as the sum of the absolute values of the eigenvalues of the matching polynomial of the graph $G_{n}$. For the Erdös-Rényi type random graph $G_{n, p}$ of order $n$ with a fixed probability p, where p is a real number greater than zero and less than 1 , that is, the graph $\mathrm{G}$ on $\mathrm{n}$ vertices by connecting two vertices with probability $\mathrm{p}(\mathrm{e})$, and each edge is independent of other one. Chen, Li and Lian solved a conjecture proposed by Gutman and Wagner, that is, the expectation of the matching energy of $G_{n, p}$ converges to a certain number associated with $\mathrm{n}$ and $\mathrm{p}$ almost surely. But they only did the result for random bipartite graphs. In this paper, we give some lower bounds for the matching energy of random bipartite graphs. And then we will use Chen et al's method to generalize this conclusion to any random multipartite graphs.
\end{abstract}

Keywords: Random Graphs, Matching Energy, Multipartite Graphs

\section{Introduction}

Let $G$ be a simple graph with vertex set $V(G)$ and edge set $E(G)$. And its vertex number and edge number are denoted by $|V(G)|$ and $|E(G)|$, respectively. A matching of $G$ is a set of independent edges in $G$, and a $k$-matching of $G$ is a matching of $G$ that has exactly $k$ edges, denoted by $M(G, k)$. Let $m(G$, $k$ ) be the number of $k$-matchings, i.e., the number of selections of $k$ independent edges, or the number of $k$-element independent edge sets of a graph $G$. Specifically, $m(G, 1)=m$ and $m(G, k)=0$ for $k>\frac{n}{2}$. It is both consistent and convenient to set $m(G, 0)=1$. The matching polynomial of a graph $G$ of order $n$ is defined as

$$
\alpha(\mathrm{G})=\alpha(\mathrm{G}, \lambda)=\sum_{k \geq 0}(-1)^{k} m(G, k) \lambda^{n-2 k} .
$$

The matching polynomial has many important implications in statistical physics and chemistry; see [6, 9, 14]. Many results on the properties of the matching roots have been obtained; see [4, 10-13].
On the other hand, the matching energy of a graph $G$ was introduced by Gutman and Wagner [5], which is defined as the sum of the absolute values of the roots of the matching polynomial of $\mathrm{G}$ (see Eq. (3)). Moreover, Gutman and Wagner found that the matching energy is a quantity of relevance with chemical applications [5] and they arrived at the relation

$$
\operatorname{TRE}(\mathrm{G})=\mathrm{E}(\mathrm{G})-\mathrm{ME}(\mathrm{G})
$$

where $\operatorname{TRE}(G)$ is the topological resonance energy of the graph $G$, and $E(G)$ is the energy of the graph $G$, i.e., the sum of the absolute values of the roots of the matching polynomial of $G$. For more information about the applications of matching energy, we refer to [6, 9]. For terminology and notation not given here, we refer to [1].

Defifinition 1 [5]. Let $G$ be a simple graph, and let $\mu_{1}, \mu_{2}, \cdots, \mu_{n}$ be the zeros of its matching polynomial. Then the matching energy of $G$ is defifined as

$$
\mathrm{ME}(\mathrm{G})=\sum_{i=1}^{n}\left|\mu_{i}\right|
$$


Theorem 2 [5]. Let $G$ be a simple graph. The matching energy of $G$ can be expressed as the following formula

$$
M E(G)=\frac{2}{\pi} \int_{0}^{\infty} \frac{1}{x^{2}} \ln \left[\sum_{k \geq 0} m(G, k) x^{2 k}\right] d x .
$$

Then Eq. (3) could be considered as the definition of matching energy and Eq. (2) would become a theorem.

Defifinition $3[6,7]$. Let $G^{\prime}$ and $G^{\prime \prime}$ be two graphs (not necessarily with the same order). If $m\left(G^{\prime}, k\right) \geq m\left(G^{\prime \prime}, k\right)$ holds for all $k=1,2,3, \cdots$, then we write $\mathrm{G}^{\prime} \geq \mathrm{G}^{\prime \prime}$. If, in addition, for at least one value of $k, m\left(G^{\prime}, k\right)>m\left(G^{\prime \prime}, k\right)$, then we write $G^{\prime}>G^{\prime \prime}$. If both $G^{\prime} \geq G^{\prime \prime}$ and $G^{\prime \prime} \geq G^{\prime}$ hold, then we say that $G^{\prime}$ and $G^{\prime \prime}$ are matching-equivalent, and write $G^{\prime} \sim G^{\prime \prime}$. If neither $G^{\prime} \geq G^{\prime \prime}$ or $G^{\prime \prime} \geq G^{\prime}$ hold, then we say that $G^{\prime}$ and $G^{\prime \prime}$ are matching-incomparable.

Bearing in mind that Eq. (2) holds, we have

Lemma 4 [8]. If $G^{\prime}$ and $G^{\prime \prime}$ are two graphs, then

$$
\mathrm{G}^{\prime} \geq \mathrm{G}^{\prime \prime} \Rightarrow \operatorname{ME}\left(\mathrm{G}^{\prime}\right) \geq \operatorname{ME}\left(\mathrm{G}^{\prime \prime}\right),
$$

and

$$
\mathrm{G}^{\prime}>\mathrm{G}^{\prime \prime} \Rightarrow \mathrm{ME}\left(\mathrm{G}^{\prime}\right)>\mathrm{ME}\left(\mathrm{G}^{\prime \prime}\right) \text {. }
$$

Next the basic properties of the matching energy and the graphs with extremal matching energy will be introduced.

Theorem 3 [8]. If the graph $\mathrm{G}$ is a forest, then its matching energy coincides with its energy.

Theorem 4 [8]. Let $\mathrm{G}$ be a graph and e is one of its edges. Let $\mathrm{G}$-e be the subgraph obtained by deleting from $\mathrm{G}$ the edge e, but keeping all the vertices of $\mathrm{G}$. Then

$$
M E(G-e)<M E(G) .
$$

Theorem 5 [8]. Among all graphs on $\mathrm{n}$ vertices, the empty graph without edges and the complete graph $K_{n}$ have, respectively, minimum and maximum matching energy.

By theorem 4, the connected graph with minimal $M E$ must be a tree. By theorem 3 , trees have equal $E$ - and $M E$-values. The fact that, among $n$-vertex trees, the star has minimal energy was established long time ago [6]. Therefore, we have

Theorem 6 [8]. The connected graph on $n$ vertices having minimum matching energy is the star $S_{n}$.

The matching energy of the empty graph is clearly 0 , and the matching energy of the star, which equals its ordinary energy, is $\operatorname{ME}\left(S_{n}\right)=2 \sqrt{(n-1)}$. There does not seem to be a similarly simple expression for the maximum matching energy $\operatorname{ME}\left(K_{n}\right)$, but the following could be proven:

Theorem 7 [5]. The matching energy of the complete graph

$K_{n}$ is asymptotically equal to $\frac{8}{3 \pi} \cdot n^{\frac{3}{2}}$. More precisely,

$$
M E\left(K_{n}\right)=\frac{8}{3 \pi} n^{\frac{3}{2}}+O(n)
$$

Theorem 8 [5]. Let $G_{n, p}$ be a random graph of order $n$ with fixed probability $p \in(0,1)$. Then

$$
M E\left(G_{n, p}\right) \geq \frac{\sqrt{p}}{\pi} n^{\frac{3}{2}}+O(\sqrt{n} \ln n),
$$

holds asymptotically almost surely.

Theorem 9 [8]. Let $G^{\prime} \cup G^{\prime \prime}$ be the graph consisting of disconnected components $G^{\prime}$ and $G^{\prime \prime}$. Then

$$
\operatorname{ME}\left(G^{\prime} \cup G^{\prime \prime}\right)=\operatorname{ME}\left(G^{\prime}\right)+\operatorname{ME}\left(G^{\prime \prime}\right) \text {. }
$$

Proposition 1. [8]. Let $F^{\prime}$ and $F^{\prime \prime}$ be two forests. Then

$$
F^{\prime} \geq F^{\prime \prime} \Rightarrow E\left(F^{\prime}\right) \geq E\left(F^{\prime \prime}\right) \text {, }
$$

and

$$
F^{\prime}>F^{\prime \prime} \Rightarrow E\left(F^{\prime}\right)>E\left(F^{\prime \prime}\right)
$$

We can directly extend Proposition 1 into:

Theorem 10 [8]. If $G^{\prime}$ and $G^{\prime \prime}$ be two forests. Then

$$
G^{\prime} \geq G^{\prime \prime} \Rightarrow M E\left(G^{\prime}\right) \geq \operatorname{ME}\left(G^{\prime \prime}\right),
$$

and

$$
G^{\prime}>G^{\prime \prime} \Rightarrow M E\left(G^{\prime}\right)>\operatorname{ME}\left(G^{\prime \prime}\right)
$$

Theorem 11 [5]. The bipartite graph on $n$ vertices having maximum matching energy is $K_{\left.\left\lfloor\frac{n}{2}\right\rfloor, \mid \frac{n}{2}\right\rceil}$.

In the 1980s the present author established a number of relations of the type $G>H$ [15-19].

Lemma 5 (Sliding). Let $G$ be a connected graph with at least two vertices, and let $u$ be one of its vertices. Denote by $P(n, k, G, u)$ the graph obtained by identifying $u$ with the vertex $v_{k}$ of a simple path $v_{1}, v_{2}, \ldots, v_{n}$. Write $n=4 p+i, i \in\{1,2,3,4\}$, and $l=\lfloor(i-1) / 2\rfloor$. Then the inequalities

$$
\begin{gathered}
\operatorname{ME}(P(n, 2, G, u)<M E(p(n, 4, G, u))<\cdots< \\
\operatorname{ME}(p(n, 2 p+2 l, G, u))<\operatorname{ME}(P(n, 2 p+1, G, u)) \\
<\cdots<M E(P(n, 3, G, u))<\operatorname{ME}(P(n, 1, G, u))
\end{gathered}
$$

hold.

Lemma 6 (Ironing). Suppose that $G$ is a connected graph and $T$ is an induced subgraph of $G$ such that $T$ is a tree and $T$ is connected to the rest of $\mathrm{G}$ only by a cut vertex $v$. If $\mathrm{T}$ is replaced by a star of the same order, centered at $v$, then the matching energy decreases (unless $T$ is already such a star). If $T$ is replaced by a path, with one end at $v$, then the matching energy increases (unless $T$ is already such a path).

Based on Lemmas 5 and 6, it was possible to characterize unicyclic, bicyclic, and tricyclic connected graphs with 
smallest and greatest matching energy. The consideration of trees will be skipped since, by theorem 3 it reduces to the well known case of extremal energy.

Denote by $U_{n}$ the set of all connected unicyclic graphs on $n$ vertices. Let $C_{n}$ be the $n$-vertex cycle, and let $S_{n}^{+}$be the graph obtained by adding a new edge to the star $S_{n}$. of course, $C_{n}, S_{n}^{+} \in U_{n}$.

Theorem 12 [5]. If $G \in U_{n} \backslash\left\{S_{n}^{+}, C_{n}\right\}$, then

$$
\operatorname{ME}\left(S_{n}^{+}\right)<M E(G)<M E\left(C_{n}\right)
$$

Denote by $B_{n}$ the set of all connected bicyclic graphs on $n$ vertices. Let $S_{n}^{*}$ be the graph obtained by adding two new edges to the same (pendent) vertex of the star $S_{n}$.

Theorem $13[5,16]$. If $G \in B_{n} \backslash\left\{S_{n}^{*}, B_{n}\right\}$, then for $n \neq 9$,

$$
\operatorname{ME}\left(S_{n}^{*}\right)<M E(G)<M E\left(B_{n}\right) .
$$

If $n=9$ and $G \in B_{9} \backslash\left\{S_{9}^{*}, B_{9}^{\prime}, B_{9}^{\prime \prime}\right\}$, then

$$
\operatorname{ME}\left(S_{9}^{*}\right)<M E(G)<M E\left(B_{9}^{\prime}\right)=\operatorname{ME}\left(B_{9}^{\prime \prime}\right) .
$$

Denote by $T C_{n}$ the set of all connected tricyclic graphs on $n$ vertices. Let $S_{n}^{\bullet}$ be the graph obtained by adding three new edges to the same (pendent) vertex of the star $S_{n}$.

Theorem 14 [17]. If $G \in T C_{n} \backslash\left\{S_{n}^{\bullet}, T C_{n}\right\}$, then for $n \neq 9$,

$$
\operatorname{ME}\left(S_{n}^{\bullet}\right)<M E(G)<M E\left(T C_{n}\right) .
$$

If $n=9$ and $G \in T C_{9} \backslash\left\{S_{9}^{\bullet}, T C_{9}^{\prime}, T C_{9}^{\prime \prime}\right\}$, then

$$
\operatorname{ME}\left(S_{9}^{\bullet}\right)<M E(G)<M E\left(T C_{9}^{\prime}\right)=\operatorname{ME}\left(T C_{9}^{\prime \prime}\right) .
$$

In 1950s, Erd $\ddot{o}$ s-R e' nyi [3] founded the theory of random graphs. The Erd $\ddot{o}$ s-R e' nyi random graph model $G_{n}(p)$ consists of all graphs on $n$ vertices in which the edges are chosen independently with probability $p$, where $p$ is a constant with $0<p<1$.

Denote by $K_{n ; \beta_{1}, \cdots, \beta_{k}}$ the complete $k$-partite graph on $n$ vertices with vertex set $V$, whose parts are $V_{1}, V_{2}, \cdots, V_{k} \quad(2 \leq$ $k=k(n) \leq n)$ satisfying $\left|V_{i}\right|=n \beta_{i}=n \beta_{i}(n), i=1,2, \cdots, k$.

The random $k$-partite graph model $G_{n ; \beta_{1}, \cdots, \beta_{k}}(p)$ consists of all random $k$-partite graphs in which the edges are chosen independently with probability $p$ from the set of edges of $K_{n ; \beta_{1}, \cdots, \beta_{k}}$. Note that $\sum_{i=1}^{k} \beta_{i}=1$ and $\beta_{i} \geq 0$ for $i=1,2, \cdots, k$. Denote by $A_{n, k}=A\left(G_{n ; \beta_{1}, \beta_{2}, \cdots, \beta_{k}}\right)=\left(x_{i j}\right)_{n \times n}$ the adjacency matrix of the random $k$-partite graph
$G_{n ; \beta_{1}, \cdots, \beta_{k}} \in G_{n ; \beta_{1}, \cdots, \beta_{k}}(p)$, where $x_{i j}$ is a random indicator variable for $v_{i} v_{j}$ being an edge with probability $p$, for $i \in V_{l}$ and $j \in V \backslash V_{l}, i \neq j, 1 \leq l \leq k$. Then $A_{n, k}$ satisfies the following properties:

(i) $x_{i j}{ }^{\prime} s, 1 \leq i<j \leq n$, are independent random variables with $x_{i j}=x_{j i}$;

(ii) $\operatorname{Pr}\left(x_{i j}=1\right)=1-\operatorname{Pr}\left(x_{i j}=0\right)=p \quad$, if $\quad i \in V_{l} \quad$ and $j \in V \backslash V_{l}$, while $\operatorname{Pr}\left(x_{i j}=0\right)=1=1$ if $i \in V_{l}$ and $j \in V_{l}, l \leq$ $l \leq k$.

Note that when $k=n, G_{n ; \beta_{1}, \cdots, \beta_{k}}=G_{n}(p)$, that is, the random multipartite graph model can be viewed as a generalization to the Erd $\ddot{o}$ s-R e' nyi model.

Chen, Li and Lian in [2] solved a conjecture proposed by Gutman and Wagner, which is now a result stated as follows.

Theorem 15 [2]. For $p \in(0,1)$, the matching energy $\operatorname{ME}\left(G_{n, p}\right)$ of the random graph $G_{n, p}$ enjoys asymptotically almost surely the following equation:

$$
\operatorname{ME}\left(G_{n, p}\right)=n^{\frac{3}{2}} p^{\frac{1}{2}}\left(\frac{8}{3 \pi}+o(1)\right) .
$$

In this paper, we give some lower bounds for the matching energy of random bipartite graphs. And then we will use Chen et al's method to generalize this conclusion to any random multipartite graphs, and obtain some lower bounds for the matching energy of random multipartite graphs.

\section{The Matching Energy of Random Bipartite Graphs}

In this section, we will give a lower bound for the matching energy of random bipartite graphs.

Theorem 16. Let $G_{1}$ and $G_{2}$ be two graphs with the same number of vertices (see Figure 1), and let $G_{1}$ be connected and bipartite with $V\left(G_{1}\right)=A \cup B$ and $G_{2}$ be disconnected with two connected components $G_{2}^{1}$ and $G_{2}^{2}$, i.e., $G_{2}=G_{2}^{1} \cup G_{2}^{2}$. Let $|A|=\left|V\left(G_{2}^{1}\right)\right|$ and $|B|=\left|V\left(G_{2}^{2}\right)\right|$. If $G$ is a graph such that $V(G)=V\left(G_{1}\right)=V\left(G_{2}\right)$ and $E(G)=E\left(G_{1}\right) \cup E\left(G_{2}\right)$, then $M E(G) \leq M E\left(G_{1} \cup G_{2}\right)$.

Proof. For any nonnegative integer $k$, we claim that

$$
m(G, k) \leq m\left(G_{1} \cup G_{2}, k\right) .
$$

We distinguish the following cases.

Case 1. All the $k$ independent edges in $M(G, k)$ appear in $G_{1}$, that is, $M(G, k)=M\left(G_{1}, k\right)$.

Case 2. All the $k$ independent edges in $M(G, k)$ appear in $G_{2}$, that is, $M(G, k)=M\left(G_{2}, k\right)$.

Case 3. Some of the $k$ independent edges in $M(G, k)$ appear in $G_{1}$, and the others appear in $G_{2}$. 
In other words, any set of $k$-matchings of $G$ is also a one of $G_{1} \cup G_{2}$. Thus, we have $m(G, k) \leq m\left(G_{1} \cup G_{2}, k\right)$. From Lemma 4, it follows that $M E(G) \leq M E\left(G_{1} \cup G_{2}\right)$.

Theorem 17. Let $G_{n ; \beta_{1}, \beta_{2}} \in G_{n ; \beta_{1}, \beta_{2}}(p)$ be a random bipartite graph. Then

$$
n^{\frac{3}{2}} p^{\frac{1}{2}}\left(\frac{8}{3 \pi}+o(1)\right)\left(1-{\beta_{1}}^{\frac{3}{2}}-\beta_{2}^{\frac{3}{2}}\right) \leq M E\left(G_{n ; \beta_{1}, \beta_{2}}\right)
$$

Proof. Note that $n=n \beta_{1}+n \beta_{2}$. Let $G_{n} \in G_{n}(P)$ be a random graph, and set $G_{n ; \beta_{1}, \beta_{2}} \subseteq G_{n}$, that is, $G_{n ; \beta_{1}, \beta 2}$ is a spanning bipartite subgraph of $G_{n}$. Then by Theorem 16,

$G_{n}, G_{n ; \beta_{1}, \beta 2}$ and $G_{n \beta_{1}} \cup G_{n \beta_{2}}$ can be seen as $G, G_{1}$ and $G_{2}$, respectively. Thus, we have

$$
\begin{aligned}
& M E\left(G_{n}\right) \leq M E\left(G_{n ; \beta_{1}, \beta_{2}} \cup G_{n \beta_{1}} \cup G_{n \beta_{2}}\right), \\
= & M E\left(G_{n ; \beta_{1}, \beta_{2}}\right)+\operatorname{ME}\left(G_{n \beta_{1}}\right)+\operatorname{ME}\left(G_{n \beta_{2}}\right) .
\end{aligned}
$$

From above, we can get

$$
\operatorname{ME}\left(G_{n}\right)-\operatorname{ME}\left(G_{n \beta_{1}}\right)-\operatorname{ME}\left(G_{n \beta_{2}}\right) \leq \operatorname{ME}\left(G_{n ; \beta_{1}, \beta_{2}}\right) .
$$

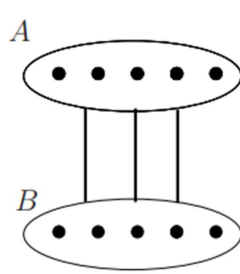

$G_{1}$

Figure 1. The separation on random bipartite graph.

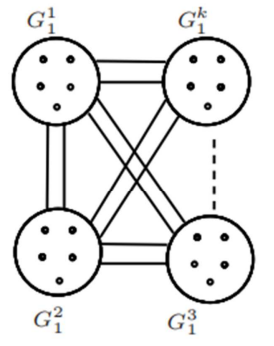

$G_{1}$
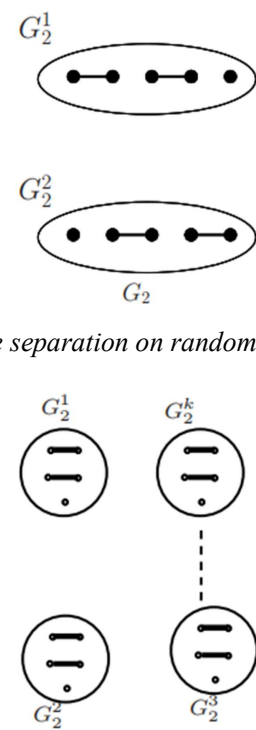

$G_{2}$

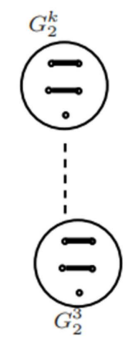

$G_{2}$

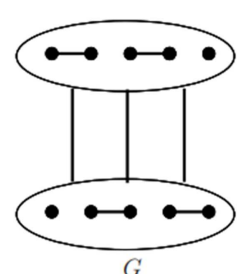

G
Figure 2. The separation on random multipartite graph.

From Theorem 15, it follows that

$$
n^{\frac{3}{2}} p^{\frac{1}{2}}\left(\frac{8}{3 \pi}+o(1)\right)\left(1-\beta_{1}^{\frac{3}{2}}-\beta_{2}^{\frac{3}{2}}\right) \leq M E\left(G_{n ; \beta_{1}, \beta_{2}}\right) .
$$

\section{The Matching Energy of Random Multipartite Graphs}

Next, we will give a lower bound for the matching energy of random multipartite graphs.
Theorem 18. Let $G_{1}$ and $G_{2}$ be two graphs with the same number of vertices (see Figure 2), and let $G_{1}$ be connected and k-partite with $V\left(G_{1}\right)=V\left(G_{1}^{1}\right) \cup V\left(G_{1}^{2}\right) \cup \cdots \cup V\left(G_{1}^{k}\right) \quad$ and $G_{2}$ be disconnected with $k$ connected components $G_{2}^{1}, G_{2}^{2}, \cdots, G_{2}^{k}$, i.e., $G_{2}=G_{2}^{1} \cup G_{2}^{2} \cup \cdots \cup G_{2}^{k}$. Let $\left|V\left(G_{1}^{i}\right)\right|=\left|V\left(G_{2}^{i}\right)\right|, \quad i=$ $1, \cdots k$. If $G$ is a graph such that $V(G)=V\left(G_{1}\right)=V\left(G_{2}\right)$ and $E(G)=E\left(G_{1}\right) \cup E\left(G_{2}\right)$, then $M E(G) \leq M E\left(G_{1} \cup G_{2}\right)$.

Proof. Similar to the proof of Theorem 16, for any non-negative integer $k$, we claim that $m(G, k) \leq m\left(G_{1} \cup G_{2}, k\right)$. We also need to consider the following cases.

Case 1. All the $k$ independent edges in $M(G, k)$ appear in $G_{1}$, that is, $M(G, k)=M\left(G_{1}, k\right)$.

Case 2. All the $k$ independent edges in $M(G, k)$ appear in $G_{2}$, that is, $M(G, k)=M\left(G_{2}, k\right)$.

Case 3. Some of the $k$ independent edges in $M(G, k)$ appear in $G_{1}$, and the others appear in $G_{2}$.

Then we can see that a set of $k$-matchings of $G$ is also a one of $G_{1} \cup G_{2}$. Thus, we have $m(G, k) \leq m\left(G_{1} \cup G_{2}, k\right)$. From Lemma 4, it follows that $M E(G) \leq M E\left(G_{1} \cup G_{2}\right)$.

Theorem 19. Let $G_{n ; \beta_{1}, \beta_{2}, \cdots, \beta_{k}} \in G_{n ; \beta_{1}, \beta_{2}, \cdots, \beta_{k}}(P)$ be a random multipartite graph. Then

$$
n^{\frac{3}{2}} p^{\frac{1}{2}}\left(\frac{8}{3 \pi}+o(1)\right)\left(1-\beta_{1}^{\frac{3}{2}}-\beta_{2}^{\frac{3}{2}}-\cdots-\beta_{k}^{\frac{3}{2}}\right) \leq M E\left(G_{n ; \beta_{1}, \beta_{2}, \cdots, \beta_{k}}\right) .
$$

Proof. It is easy to see that $n=n \beta_{1}+n \beta_{2}+\cdots+n \beta_{k}$. Let $G_{n} \in G_{n}(p)$ be a random graph, and set $G_{n ; \beta_{1}, \beta_{2}, \cdots, \beta_{k}} \subseteq G_{n}$, that is, $G_{n ; \beta_{1}, \beta_{2}, \cdots, \beta_{k}}$ is a spanning $k$-bipartite subgraph of $G_{n}$. Then by Theorem 18, $G_{n}, G_{n ; \beta_{1}, \beta_{2}, \cdots, \beta_{k}}$ and $G_{n \beta_{1}} \cup G_{n \beta_{2}} \cup \cdots \cup G_{n \beta_{k}}$ can be seen as $G, G_{1}$ and $G_{2}$, respectively. Thus, we have

$$
\begin{aligned}
& M E\left(G_{n}\right) \leq \operatorname{ME}\left(G_{n ; \beta_{1}, \beta_{2}, \cdots, \beta_{k}} \cup G_{n \beta_{1}} \cup G_{n \beta_{2}} \cup \cdots \cup G_{n \beta_{k}}\right), \\
= & M E\left(G_{n ; \beta_{1}, \beta_{2}, \cdots, \beta_{k}}\right)+\operatorname{ME}\left(G_{n \beta_{1}}\right)+\operatorname{ME}\left(G_{n \beta_{2}}\right)+\cdots+\operatorname{ME}\left(G_{n \beta_{k}}\right) .
\end{aligned}
$$

From above, we can get

$$
\begin{aligned}
& \operatorname{ME}\left(G_{n}\right)-\operatorname{ME}\left(G_{n \beta_{1}}\right)-\operatorname{ME}\left(G_{n \beta_{2}}\right)-\cdots-M E\left(G_{n \beta_{k}}\right) . \\
& \leq \operatorname{ME}\left(G_{n ; \beta_{1}, \beta_{2}, \cdots, \beta_{k}}\right) .
\end{aligned}
$$

From Theorem 15, it follows that

$$
\begin{aligned}
& n^{\frac{3}{2}} p^{\frac{1}{2}}\left(\frac{8}{3 \pi}+o(1)\right)\left(1-\beta_{1}^{\frac{3}{2}}-\beta_{2}^{\frac{3}{2}}-\cdots-\beta_{k}^{\frac{3}{2}}\right) \\
& \leq M E\left(G_{n ; \beta_{1}, \beta_{2}, \cdots, \beta_{k}}\right)
\end{aligned}
$$




\section{Conclusion}

From the above discussion, we obtain the lower bounds for the matching energy of random bipartite graphs, that is, if $G_{n ; \beta_{1}, \beta_{2}} \in G_{n ; \beta_{1}, \beta_{2}}(p)$ be a random bipartite graph, then we have

$$
n^{\frac{3}{2}} p^{\frac{1}{2}}\left(\frac{8}{3 \pi}+o(1)\right)\left(1-\beta_{1}^{\frac{3}{2}}-\beta_{2}^{\frac{3}{2}}\right) \leq M E\left(G_{n ; \beta_{1}, \beta_{2}}\right) .
$$

We use the same method to generalize the conclusion to the random multipartite graphs, and we give the lower bounds for the matching energy of the random multipartite graphs, which is for any random multipartite graph $G_{n ; \beta_{1}, \beta_{2}, \cdots, \beta_{k}} \in G_{n ; \beta_{1}, \beta_{2}, \cdots, \beta_{k}}(P)$. Then we obtain

$$
n^{\frac{3}{2}} p^{\frac{1}{2}}\left(\frac{8}{3 \pi}+o(1)\right)\left(1-\beta_{1}^{\frac{3}{2}}-\beta_{2}^{\frac{3}{2}}-\cdots-\beta_{k}^{\frac{3}{2}}\right) \leq M E\left(G_{n ; \beta_{1}, \beta_{2}, \cdots, \beta_{k}}\right) .
$$

\section{Acknowledgements}

Supported by the NSFQH No. 2018-ZJ-925Q; NSFC No. 11701311; NSFGD No. 2016A030310307.

\section{References}

[1] J. A. Bondy, U. S. R. Murty, Graph Theory, GTM 244, Springer, 2008.

[2] X. Chen, X. Li, H. Lian, The matching energy of random graphs, Discrete Applied Mathematics 193 (2015), 102-109.

[3] P. Erdös, A. Rényi, On random graphs I, Publ. Math. Debrecen 6 (1959), 290-297.

[4] E. J. Farrell, An introduction to matching polynomial, J. Combin. Theory Ser. B 27 (1979), 75-86.

[5] I. Gutman, S. Wagner, The matching energy of a graph, Discr. Appl. Math. 160 (2012), 2177-2187.
[6] I. Gutman, Acyclic systems with extremal H“uckel $\pi$-electron energy, Theor. Chim. Acta. 45 (1977), 79-87.

[7] I. Gutman, Partial ordering of forests according to their characteristic polynomials, in: A. Hajnal, V. T. Sos (Eds.), Combinatorics, North-Holland, Amsterdam, 1978, pp. 429436.

[8] I. Gutman, X. Li, Energies of Graphs - Theory and Applications, Math. Chem. Monogr. Vol. 17 (2016). ISBN 978-86-6009-033-3, Kragujevac, Serbia.

[9] I. Gutman, M. Milun, N. Trinajstić, Graph theory and molecular orbitals 19, non-parametric resonance energies of arbitrary conjugated systems, J. Amer. Chem. Soc. 99 (1977), 1692-1704.

[10] I. Gutman, The matching polynomial, MATCH Commun. Math. Comput. Chem. 6 (1979), 75-91.

[11] C. Godsil, Matchings and walks in graphs, J. Graph Theory 5 (1981), 285-297.

[12] C. Godsil, I. Gutman, On the theory of the matching polynomial, J. Graph Theory 5 (1981), 137-144.

[13] C. Godsil, Algebraic Combinatorics, Chapman Hall, New York, 1993.

[14] O. J. Heilman, E. H. Lieb, Theory of monomerCdimer systems, Comm. Math. Phys. 25 (1972).

[15] I. Gutman, Graphs with greatest number of matchings, Publ. Inst. Math. (Beagrad) 27 (1980) 581-586.

[16] I. Gutman, Correction of the paper "Graphs with greatest number of matchings", Publ. Inst. Math. (Beagrad) 32 (1982) 61-63.

[17] I. Gutman, D. Cvetkovic, Finding tricyclic graphs with a maximal number of matchins-another example of computer aided research aided research in graph theory, Publ. Inst. Math. (Beagrad) 35 (1984) 33-40.

[18] I. Gutman, F. Zhang, On a quasiordering of bipartite graphs, Publ. Inst. Math. (Beagrad) 35 (1984) 33-40.

[19] I. Gutman, F. Zhang, On the ordering of graphs with respect to their matching numbers, Discr. Appl. Math. 15 (1986) 25-33. 